\title{
Effectively unpaired electrons in bipartite lattices within the generalized tight-binding approximation: application to graphene nanoflakes
}

\author{
A.V.Luzanov \\ STC "Institute for Single Crystals", National Academy of Sciences of \\ Ukraine, 60 Lenin Ave., 61001 Kharkiv, Ukraine
}

Received September 29, 2014

\begin{abstract}
A semiempirical technique is proposed for bipartite structures (lattice-like systems with two interpenetrating sublattices). For such systems, the corresponding one-electron tightbinding model can be easily modified to include electron correlation effects, although in a rough manner. It allows one to describe the so-called effectively unpaired electrons (EUE) in giant many-electron systems by using even uncomplicated hardware. The average EUE occupancy is interpreted as a counterpart of the order parameter reflecting a hidden antiferromagnetic structure of the strongly correlated system. We illustrate the developed method by analyzing EUE for several model problems (nanoflakes and nanoribbons) mimicking the graphene-based materials.
\end{abstract}

Предложен полуэмпирический метод расчета многоэлектронных систем для двудольных структур (решеточных систем с двумя взаимопроникающими подрешетками). Для таких систем соответствующая модель сильной связи может быть легко модифицирована с тем, чтобы учесть, хотя и грубо, әффекты электронной корреляции. Это позволяет описывать так называемые әффективно распаренные электроны (ЭРС) в гигантских многоэлектронных системах, применяя при этом даже скромную вычислительную технику. Средняя заселенность распаренных электронов интерпретируется как аналог параметра порядка, отражающего скрытую антиферромагнитную структуру сильно коррелированной системы.

Ефективно розпарені електрони у дводольних гратках за узагальненою схемою сильного зв'язку. Застосування до графенових наночастинок. А.В.Лузанов.

Запропоновано напівемпіричну схему розгляду багатоелектронних систем у дводольних структурах (граткоподібних системах 3 двома взаємопроникаючими підгратками). Щодо таких систем легко модифікувати одноелектронне наближення сильного зв'язку таким чином, щоб урахувати ефекти електронної кореляції, хоча й грубо. Це дозволяє описування так званих ефективно розпарених електронів (EPE) у гігантських багатоелектроних системах, використовуючи при цьому навіть нескладну обчислювальну техніку. Середню заселеність ЕРЕ потрактовано як аналог параметра порядку, що відображає приховану антиферомагнітну структуру сильно корельованої системи. Пролюстровано розвинутий метод, аналізуючи ЕРЕ декількох модельних систем (наночастинкок та нанострічок), котрі імітують нанографенові матеріали. 


\section{Introduction}

In many cases the atomic solids materials can be treated as giant covalent network structures. Such structures are frequently based on the so-called bipartite lattices. It is well known that carbon nanotubes and graphene-related materials are of this structural type. The bipartite lattices, by definition, belong to a class of lattices containing two type of atoms (sites), so that each atom has nearest neighbors of the opposite type. In other words, the bipartite lattice is formed by two interpenetrating sublattices, and each of these sublattices contains only one kind of atoms. There are many remarkable theorems for bipartite systems studied in the framework of many-body theory and related quantum chemical approaches [1-6]. In particular, according to Konig's simple theorem, plane bipartite graph does not contain cycles of odd length [6].

It should be recognized that in the current computational physics, multifarious assumptions and approaches are applied for modeling bipartite lattices and related systems [7]. Some of them employ the sophisticated ab initio machinery which provides the most accurate results. However, it is too demanding and has a restricted field of applications. By this reason, simple and even rough approaches often get involved when studying too complex structures. Among the basic approaches, the tight-binding (TB) model and its reasonably simple extensions did not lose a significance as a useful tool for semi-quantitative description [8-11]. However, TB in its nature is essentially the one-electron theory, that is, no electron correlation effects are explicitly taken into account when using the TB model. At the same time, many principal properties are determined by electron correlation, and the problem is how to include, even partly, important features of electron correlation theories in the original TB model without sophisticating it. One possible way was previously outlined in [12] where we studied the magnetic field effects within the Huckel model (which is in fact the TB scheme for $\pi$-electrons in the unsaturated hydrocarbons.

Our objective here is to extend the Huckel-like correlation model of [12] to the above-mentioned network structures. The primary focus of the present study is on the characterization of the diamagnetic materials by the so-called effectively unpaired electrons (EUE) [13-15]. The latter appear even in any covalent structures with strong electron correlation, and the EUE issues have become one of the foci and points of current interest [16-19]. As a starting point, we make use of a simplified version of the unrestricted Hartree-Fock (UHF) model, or in other words, the spin-polarized model of electron correlation. There are many papers concerning the main features of the UHF theory and ensuing practical techniques [20-26]. In the present context, specific relations of Brickstock and Pople [2] for bipartite systems within UHF are the key for the rationale of our simplified scheme.

The plan of the paper is following. In the next section we sketch the EUE theory in the form which is more appropriate for our aims. In section 3 some known relations for bipartite lattices in the TB approximation are given. Then the extension of TB to a simplistic electron-correlation treatment and EUE issues is given (section 4). Testing and applications of the proposed scheme (mainly to conjugated polymers and graphene nanoclusters) are presented in sections 5 and 6 , respectively. Finally, we sum up the main points of the paper (section 7 ).

\section{Effectively unpaired electrons in a nutshell}

In this section, we give a brief outline of the EUE theory for ground electronic states which, for the diamagnetic molecules, are naturally the singlet states. It should be emphasized that evidently there exist no spin density effects in diamagneric molecule. Hence, the electrons being 'effectively' unpaired (e.g., in the dissociated hydrogen molecule or in radical pairs) remain, as a whole, in the singlet state due to the total spin conservation law. This fact indicates some type of correlation between the particles, particularly between the spatially separated ones, as it is in the classical EinsteinPodolsky-Rosen pair. At the same time, systems with a large degree of electron unpairing become singlet biradicaloids or even polyradicals which possess many unusual properties.

In quantum chemistry terms, the EUE problem is formulated for arbitrary singlet states as follows. Let $D$ is a spin-free oneelectron density matrix (charge density matrix) of the $N$-electron state under study. For singlet states with slight electron correlation effects one can employ the usual independent-particle description, that is the restricted Hartree-Fock (RHF) approach. In 
this case we have the corresponding $D$ matrix with precisely $n=N / 2$ nonzero eigenvalues $\left\{\lambda_{i}\right\}$, all equal to 2 . Generally, the eigenvalues, $\left\{\lambda_{i}\right\}$, of $D$ are termed as the natural orbital occupation numbers (NOON). Clearly, the NOON value 2 is related to typical non-correlated electron pairs (as in usual textbook formulations of the Pauli principle). It means that for the such states all orbitals are occupied twice:

$$
D=2 \sum_{i \leq n}\left|\varphi_{i}\right\rangle\left\langle\varphi_{i}\right| .
$$

In the case of the correlated singlet states, deviation of the NOON spectrum from 2 properly characterizes EUE.

There are several numerical measures (denoted usually by $N_{e f f}$ ) for quantifying EUE and describing associated characteristics (e.g., EUE distributions) [13]. We will follow the EUE measure due to HeadGordon [14] since for typical singlet states a similar measure has a clear hole-particle meaning as established in $[15,18]$. In this hole-particle interpretation we have the expression

$$
N_{e f f}=2 \sum_{a>n} \lambda_{a}
$$

where NOONs $\left\{\lambda_{a}\right\}$ are related to "virtual" (particle) one-electron states which are occupied owing to electron correlation. Along with $N_{\text {eff }}$ the EUE density matrix, $D^{E U E}$, can be easily derived in the form of the spectral representation

$$
D^{E U E}=\sum_{i \leq n}\left(2-\lambda_{i}\right)\left|\varphi_{i}\right\rangle\left\langle\varphi_{i}\left|+\sum_{a>n} \lambda_{a}\right| \varphi_{a}\right\rangle\left\langle\varphi_{a}\right| .
$$

Here $\left|\varphi_{a}\right\rangle$ and $\left|\varphi_{i}\right\rangle$ are the eigenvectors of $D$ (natural orbitals); they are assigned to the virtual orbital and occupied orbital subspaces, respectively. Notice that numbers $2-\lambda_{i}$ correspond to hole occupancies. It can be proved that $D^{E U E}$ is properly normalized: $\operatorname{Tr} D^{E U E}=N_{\text {eff }} \cdot$

In practice, more tractable expressions can be used for handling EUE within typical many-electron approaches. For instance, within UHF we have

$$
D=\rho_{\alpha}+\rho_{\beta},
$$

with $\rho_{\alpha}$ and $\rho_{\beta}$ being the conventional UHF density matrices. They are simply the oneelectron projectors onto occupied spin-up and spin-down orbitals, respectively. In accordance with this, we have

$$
N_{e f f}=N-2 \sum_{i \leq n} \lambda[\alpha \beta],
$$

where $\lambda_{i}^{[\alpha \beta]}$ is the squared root from the eigenvalue of $\rho_{\alpha} \rho_{\beta}$. Other details can be found in $[14,18]$. Obviously, we return to RHF, when putting $\rho_{\alpha}=\rho_{\beta}$, so $D_{0}$ is the same as in Eq. (1), and $N_{\text {eff }}=0$.

\section{Tight-binding model for bipartite lattices}

Before presenting a suitably generalized TB approximations we need some simple facts about the customary TB scheme for bipartite systems. For this, it is necessary to write down the standard TB Hamiltonian $h^{0}$ in the atomic orbital representation. Recall that we deal with the structures which are made of atoms of the same kind (the carbon atoms in all our applications). Notice also that as in [5], we make use of the term "lattice" in an extended meaning allowing it for finite lattices and even for any finitesize atomic structures. In our specific problem the standard atomic basis set $\left\{\left|\chi_{\mu}\right\rangle\right\}$ is employed (it is comprised of the orthonormalized $2 p_{z}$-orbitals assigned to each carbon atom). With this basis set we have

$$
h_{0}=\sum_{\mu, v} \beta_{\mu \nu}\left|\chi_{\mu}\right\rangle\left\langle\chi_{v}\right|
$$

Here and elsewhere $\beta_{\mu \nu} \equiv \beta_{0}$ is the usual hopping integral (if $\mu \neq v$ ), or resonance integral, between nearest-neighbor sites $\mu$ and $v$, and moreover, diagonal elements $\beta_{\mu \mu} \equiv 0$. It is convenient to measure all energies in units of $\left|\beta_{0}\right|$ (the $\beta_{0}$ value is normally negative). Further, in the case of bipartite lattices we can always renumber lattice sites in such a way that the TB Hamiltonian matrix, $h^{0}$, in the atomic orbital basis can be cast into the following block form:

$$
h_{0}=-\left(\begin{array}{cc}
0 & B \\
B^{+} & 0
\end{array}\right) .
$$

Here $B$ is a bond matrix, or in graph theory terms, biadjacency matrix: $B_{\mu \nu}=1$ if $\mu$ and $\nu$ are nearest-neighbor sites, otherwise $B_{\mu \nu}=0$.

From Eq. (7) a variety of theorems and useful results can be deduced. One of them is due to G.G.Hall [27]. For completeness, we recall that in standard terms the $D$ ma- 
trix in AO representation is the conventional charge and bond-order matrix (e.g., see [21], p.139). In the case of the Huckel (TB) approximation we will denote this $D$ matrix by $D_{0}$. In fact, $D_{0}$ is a specific case of a more general expression in Eq. (3), when $\rho_{\alpha}=\rho_{\beta} \equiv \rho_{0}$, and matrix $\rho_{0}=\left(\rho_{0}\right)^{2}$ projects onto the usual doubly occupied MOs, so $D_{0}=2 \rho_{0}$. Hall's elegant result for bipartite systems is

$$
D_{0}=\left(\begin{array}{cc}
I & B\left(B^{+} B\right)^{-1 / 2} \\
\left(B^{+} B\right)^{-1 / 2} B^{+} & I
\end{array}\right),
$$

that is, bond orders (off-diagonal elements of $D_{0}$ ) are immediately determined by biadjacency matrix $B$. It can be straightforwardly derived from the known relationship $D_{0}=I-h^{0} /\left|h^{0}\right|$, where $\left|h^{0}\right|=\left[\left(h^{0}\right)^{2}\right]^{1 / 2}$ (see $[28,29])$. The numerically stable methods for direct computations of such matrix functions (avoiding matrix diagonalization) are given in [29,30]. And again, owing to $D_{0}=2 \rho_{0}$ and $\rho_{0}=\left(\rho_{0}\right)^{2}$, the above $D_{0}$ obeys the spectral resolution (1), that is the EUE effects are surely not possible within the conventional Huckel TB approximation.

\section{Modifying tight-binding model for unpairing electrons}

Now we will describe the previously mentioned Huckel-like correlation model [12] in more detail, and generalize our results to a larger context. It allows us to extend TB to strongly correlated electronic materials with bipartite lattice geometries (including alternant hydrocarbons in organic chemistry). Hereafter, we will term our approach as the quasi-correlated tight-binding (QCTB) model. The model is a very simplified, and yet specifically modified, version of the conventional UHF scheme. Notice that the UHF problem is significantly reduced for bipartite systems because of the restrictions imposed by a special structure of the matrices involved. Really, in the UHF case one must simultaneously solve two coupled eigenvalue problems - for the Fock matrices $f^{\alpha}$ and $f^{\beta}$ associated with spin-up and spin-down one-electron states. These Fock matrices have a block structure more complicated than in Eq. (7):

$$
f^{\alpha}=-\left(\begin{array}{cc}
A & \tilde{B} \\
\tilde{B}^{+} & C \\
-A & \tilde{B} \\
\tilde{B}^{+} & -C
\end{array}\right),
$$

where $\tilde{\beta}$ is a slightly modified previous matrix $B$, and now non-null diagonal blocks $A$ and $C$ introduce additional intersite effects. All the blocks in Eq. (10) now depend on $\rho_{\alpha}$ and $\rho_{\beta}$ (see the last two equations in [2]). However, qualitative reasoning and numerical examples tell us that $\tilde{B}$ is not essentially different from $B$, and off-diagonal elements of $A$ and $C$ give mainly the second order effects. Thus, in addition to $B$, the most important are diagonal matrix elements $A_{\mu \mu}$ and $C_{\mu \mu}$ in $A$ and $C$, respectively. All $A_{\mu \mu}$ can be roughly putted the same constant value, say $\delta$, and, analogously, $C_{\mu \mu}=-\delta$. With this, $\delta$ is treated as a fitting parameter. As a result, we arrive at the effective Hamiltonians matrices

$$
f^{\alpha}=h^{\delta}, \quad f^{\beta}=h^{-\delta},
$$

where a new Huckel Hamiltonian $h^{\delta}$ is of the form

$$
h^{\delta}==-\left(\begin{array}{cc}
\delta I & B \\
B^{+} & -\delta I
\end{array}\right) .
$$

This Hamiltonian has previously appeared in the usual (that is, correlationfree) Huckel theory for describing systems with two different types of sites in bipartite-like lattices (e. g., in the hexagonal boron nitride "white graphite"). Some general relations for such systems were given in [31]. In our case, however, the meaning of $h^{\delta}$ is quite different. By the pair of effective Hamiltonians in Eq. (10) we introduce correlation effects for usual bipartite lattices with the same type of sites in lattices. On this account, $\delta$ is only an assisting parameter which artificially introduces electron correlation effects into the TB formalism. Nevertheless, formal relations from [31] are applicable and useful in the present context; for instance, we have

$$
\begin{gathered}
D_{0}^{\delta}= \\
=\left(\begin{array}{cc}
I+\delta\left(\delta^{2} I+B B^{+1 / 2}\right. & B\left(\delta^{2} I+B^{+} B\right)^{-1 / 2} \\
\left.\delta^{2} I+B^{+} B\right)^{-1 / 2} B^{+} & I-\delta\left(\delta^{2} I+B^{+} B\right)^{-1 / 2}
\end{array}\right) .
\end{gathered}
$$

which extends Eq. (8) to systems with the Huckel Hamiltonian $h^{\delta}$ from Eq. (11).

We are now in a position to elucidate the solution of the EUE problem within the QCTB model. From Eqs. (10), (12) we infer that

$$
\rho_{\alpha}=D_{0}^{\delta} / 2, \quad \rho_{\beta}=D_{0}^{-\delta} / 2 .
$$

Then, Eq. (4) is easily specified to be 


$$
D==-\left(\begin{array}{cc}
I & \left(\delta^{2} I+B^{+} B\right)^{-1 / 2} B^{+} \\
B\left(\delta^{2} I+B^{+} B\right)^{-1 / 2} & I
\end{array}\right),{ }^{(14)}
$$

and the problem of diagonalizing it is a standard one. The resulting NOON spectrum is

$$
\begin{aligned}
& \lambda_{i}^{+}=1+\varepsilon_{i} / \sqrt{\delta^{2}+\varepsilon_{i}^{2}}, \\
& \lambda_{i}^{-}=1-\varepsilon_{i} / \sqrt{\delta^{2}+\varepsilon_{i}^{2}},
\end{aligned}
$$

where nonnegative quantities $\varepsilon_{i} \equiv\left|\varepsilon_{i}\right|$ are eigenvalues of $\left(B^{+} B\right)^{1 / 2}$, that is $\left\{\varepsilon_{i}\right\}$ is the bipartite graph spectrum. The same $\left\{\varepsilon_{i}\right\}$ spectrum is precisely the Huckel energy spectrum (in modulus) which is generated by matrix (7). Thus, in accordance with Eq. (2) we have

$$
N_{\text {eff }}=2 \sum_{i=1}^{n} \lambda_{i}^{-},
$$

or explicitly:

$$
N_{e f f}=N-2 \sum_{i=1}^{n} \varepsilon_{i} / \sqrt{\delta^{2}+\varepsilon_{i}^{2}} .
$$

This simple working expression permits us to compute the main EUE measure directly from Huckel energy spectrum which is very easy to find either analytically or, most frequently, numerically.

For instance, let us consider the polyacetylene (polyene) chain $[-(\mathrm{CH}=\mathrm{CH})-]_{n}$ as a paradigmatic example of strong correlation in the physics of conjugated polymers [32,33]. Its Huckel spectrum (see any quantum chemistry textbook) is $\varepsilon_{k}=2 \cos [\pi k /(2 n+1)]$. For the case of $n \rightarrow \infty$ computations on Eq. (16) are in fact trivial, leading to

$$
N_{\text {eff }}=N\left(1-\frac{2}{\pi} \arcsin \left[1 / \sqrt{1+\delta^{2} / 4}\right]\right) .
$$

From this equation we see that in the limit of large $\delta$ (very strong correlation effects) the EUE index $N_{\text {eff }} \rightarrow N$, as it should be. Recall that in this problem $N=2 n$ is a number of carbon atoms in the polyene chain, that is the number of $\pi$-electrons, so the value $N_{\text {eff }}=N$ corresponds to breaking each of $\pi$-bonds, when all $\pi$-electrons are unpaired.

Before ending this section it is worth mentioning other approaches which have much in common with the QCTB scheme [12]. If we take the EUE issues apart, the most similar to our method is the earlier approximate one-parameter UHF theory [34] which is based on somewhat different grounds. In fact, in [34] the conventional UHF scheme within TB is also a starting point, and the bipartite structure (7) is taken account as well. At the same time, the main deficiency of the UHF theory remains untouched. To be more definite, the simplified UHF solutions of [34] exist only for systems with sufficiently strong correlation effects. This differs from our scheme in which one can always obtain nonzero correlation effects by a suitable choice of the fitting parameter $\delta$. However, for very strongly correlated systems, QCTB and the approximation scheme [34] should be virtually equivalent. Another interesting paper is [35] where, if deleting insignificant terms, the one-electron Hamiltonian matrix is introduced in the form (11) [see loc cit, Eq. (11)]. However, an analogue of $\delta$ has there indefinite origin, and in [35] no correlation effects are involved within this special one-electron TB model. Few words should be added about our parameter $\delta$. For the systems described below, we made use of $\delta=7 / 24$ (in the $\left|\beta_{0}\right|$ units). It was obtained from fitting the QCTB results to the more reliable data given in [18] (small polyacene systems, such as tetracene and pentacene, were used). If needed, one can make a similar fitting procedure for other structural classes.

\section{Testing and applying QCTB model to conjugated polymers}

It is necessary now to study the accuracy and applicability of the developed model. To this end, we performed comparable calculations of $N_{\text {eff }}$ for several typical $\pi$-conjugated hydrocarbons (see Table 1). For large systems a more suitable is the $N_{\text {eff }}$ value per electron, that is the index

$$
\bar{N}_{e f f}=N_{e f f} / N
$$

which will serve us as the main EUE characteristics. Most examples for Table 1 are taken from [18] where numerical data for several high-level approximations are given. Furthermore, additional systems were also considered, and they are included in Table 1. Judging from the previous results [18], the coupled cluster doubles (CCD) theory (with using the RHF reference determinant of UHF natural orbitals [22]) is the most reliable one among the other affordable schemes for comparably large systems 
Table 1. Comparison of the EUE index $\bar{N}_{e f f}$ calculated with the UHF, QCTB, and CCD $\pi$-electron approximations.

\begin{tabular}{|c|c|c|c||}
\hline System & UHF & QCTB & CCD \\
\hline Anthracene & $\approx 0$ & 0.046 & 0.049 \\
Tetracene & 0.044 & 0.055 & 0.055 \\
Pentacene & 0.061 & 0.062 & 0.061 \\
Hexacene & 0.070 & 0.068 & 0.065 \\
Bisanthene & 0.056 & 0.059 & 0.057 \\
Pyranthrene & 0.036 & 0.050 & 0.049 \\
Perylene & $\approx 0$ & 0.046 & 0.045 \\
Terrylene & 0.024 & 0.050 & 0.046 \\
Zetrene & 0.058 & 0.060 & 0.059 \\
Phenanthrene & $\approx 0$ & 0.036 & 0.043 \\
Picene & $\approx 0$ & 0.038 & 0.043 \\
\hline
\end{tabular}

treated in [18]. Thus, when inspecting Table 1, one must compare between the QCTB and CCD results first of all. We see that QCTB typically provides a quite satisfactory description of EUE. Moreover, QCTB also gives reasonable semiquantitative results for the systems with small electron correlation effects, where the conventional UHF methods inevitably fails (see examples with $N_{\text {eff }} \approx 0$ in Table 1).

It would be interesting as well to compare QCTB against existing ab initio schemes. Unfortunately, a direct comparison seems to be not achievable due to a lack of details in the most published works. But some principal features of different approaches can be observed. For definiteness, take such an important characteristics as the NOON spectrum which is given in many papers on the EUE problems [16-18]. In particular, the hexacene molecule and its oligomeric structures of periacene type were studied within the quite reliable averaged quadratic coupled cluster scheme with a basis set of double zeta quality [17]. The needed NOON spectra are graphically displayed in Fig. 3 of loc cit. Our plots within the QCTB scheme are presented in Table 2. From comparing these two sets of data we observe their surprisingly close resemblance (e. g., in the case of the last system, the occurrence of two $\lambda_{i}(\approx 1.02$ and $\approx 0.98-0.99$ in our plots), that is quite near 1 , and clearly corresponds to almost unpaired electrons).

We are now apply the proposed method to the generic $\pi$-conjugated polymers and simplest graphene nanoribbons (GNR). For
Table 2. The EUE index $\bar{N}_{\text {eff }}$ and discrete NOON spectrum $\left\{\lambda_{i}\right\}_{1 \leq i \leq N}$ for some structures based on hexacenene subunits. Here $N$ is the number of carbon atoms.

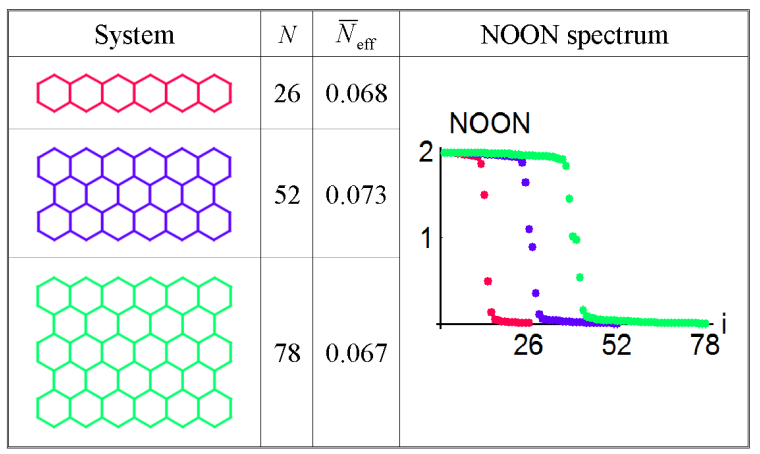

some of them the corresponding Huckel spectrum is analytically know, for other the spectrum can be computed numerically by the conventional energy band theory. We restrict our study to polyacetylenes, polyacene, 4-ZGNR (see, e.g., [36] about nomenclature of graphene nanoribbons), and poly(perianthracene). All these polymeric structures are displayed in Table 3 where we introduced the alternation parameter $\eta$. The bond length alternation in the carbon chain is normally taken into account by using hopping integrals $\beta_{\mu, \mu+1}=\left[1+(-1)^{\mu+1} \eta\right] \beta_{0}$, where $\eta$ is usually small quantity (we put $\eta=0.07$ ). Then, for the polyacetylene the Huckel spectrum, which is obtained by Lennard-Jones [37], is of the form

$$
\varepsilon_{k}=\sqrt{2}\left[1+\eta^{2}+\left(1-\eta^{2}\right) \cos \pi k\right]^{1 / 2},
$$

and $0 \leq k \leq 1$. For the polyacene (the third system in Table 3), in accordance with Coulson [38],

$$
\varepsilon_{k}=\left[1 \pm(9+8 \cos \pi k)^{1 / 2}\right] / 2,
$$

For obtaining the electronic $\pi$-band structure in the 4-ZGNR and poly(perianthracene) [the last two systems in Table 3] we employed the computer program from [39]. As seen from Table 3, for all polymer chains, except the polyacetylene with alternating bonds and poly(perianthracene) [the last structure in the Table], we find continuous spectrum covering the whole interval $[0,2]$. Particularly, in polyacene and 4-ZGNR a crowding effect is observed for the NOON spectrum near 1 . In this case, it would also be helpful to analyze the density of natural orbitals in the vicinity $\lambda_{i} \cong 1$. To do this, one can follow the technique familiar from the band theory [40]. However, 
Table 3. The $\bar{N}_{\text {eff }}$ index and continuous NOON spectrum $\lambda(k)(0 \leq k \leq 1)$ for infinite $\pi$-conjudated polymer chains.

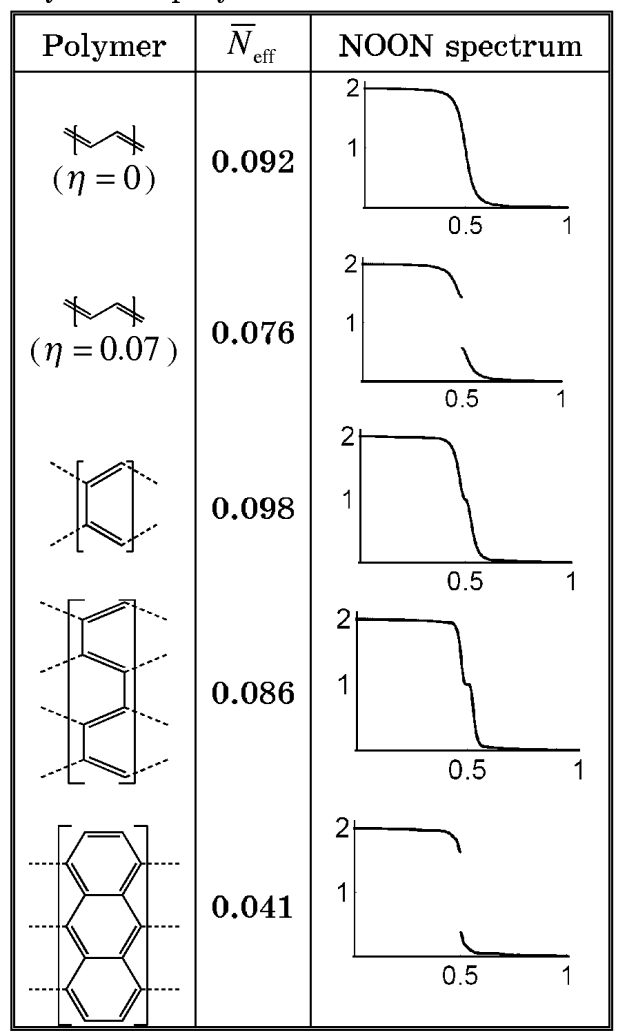

this aspect of the EUE theory is beyond the scope of the present paper. The above enhance of polyradical character in polyacene and 4-ZGNR essentially distinguishes the latter from the polyacetylene (with alternating bonds) and from poly(perianthracene). There is a gap in the NOON spectrum for them, and no crowding $\lambda_{i}$ near a "polyradical range", that is near 1 . It is interesting that significant difference in the $\bar{N}_{\text {eff }}$ index between the 4-ZGNR and poly(perianthracene) can be easily understood in terms of Clar's aromatic sextet theory (see, e.g., [41]).

The obtained results can be additional interpreted in terms of antiferromagnetism. The such terminology is frequently used when analyzing $\pi$-conjugated polymers [32, $36,41-44]$. However, the nature of antiferromagnetism for the overall singlet state is not so simple as in the case of molecular ferromagnetism [32, 42, 45]. Indeed, even a local spin density is absent in the singlet state. Hence no conventional Neel-like spin structure is possible for single molecule in its singlet state. If so, we cannot introduce as usual the antiferromagnetic order parameter (such as average difference of spin density between neighboring atoms). We as- sume that for the correlated singlet states, spin density matrix should be replaced with EUE matrix (3). Then, for polymer structures, index $\bar{N}_{\text {eff }}$ can serve as a new order parameter. This index satisfies inequality: $0 \leq \bar{N}_{e f f} \leq 1$, that is natural to expect from the order parameter (in our case $\bar{N}_{\text {eff }}=1$ corresponds to the full Neel state with the maximal "spin" value in each sublattice of the bipartite structure). The above suggested interpretation introduces an obviousness in understanding EUE for bipartite network structures. Furthermore, by adopting this reasoning, one can invoke the best spin-polarized (UHF-like) solution which may be constructed for any good-quality wave function (see [46] for the appropriate algorithm). It makes it possible to reinterpret even the exact wave function in terms of the $\bar{N}_{e f f}$ index as the order parameter which is able to characterize a hidden antiferromagnetic nature of the state under study.

\section{Unpairing in graphene nanoflakes}

As was stated in [47], "Graphene is a rapidly rising star on the horizon of materials science", and, it seems that this rise will be sustainable $[41,48-50]$. A large part of current interest in this field relates to graphene nanoribbons, nanoislands, nanowiggles and other unusual honeycomb structures [51], that is also bipartite lattices. In this section we apply QCTB to the largescale $\pi$-electron systems mimicking graphene nanoflakes (small pieces of graphene [51-55]). We start with the graphene nanoflakes having hexagonal honeycomb lattice. An initio calculations of such small-sized nanoclusters were recently performed (e.g., see $[41,52,54]$. By using the proposed, although rough, approach we are able to examine surely larger systems using easy computer software with not powerful hardware (e.g., laptops).

We restrict our study to nanoclusters with $N \sim 10^{3}$ (see Table 4 ). The first three systems in Table 1 are of the class of nanoflakes with the $D_{6 h}$ symmetry (hexagonal graphene nanoflakes). Two distinct types of the hexagonal nanoflakes are known: those having armchair edges (as in the first system in Table 4) and those having zigzag edges (as in the second and third structures in Table 4). From the displayed results we can tell that large armchair and zigzag hexagonal nanoflakes have a small difference in energy stability, but a significant difference 
Table 4. The TB energy, $\bar{E}^{0}$, and QCTB energy, $\bar{E}$, both per electron, and the EUE characteristics for the graphene nanoflakes. $\bar{N}_{\text {eff }}^{\text {bord }}$ is analogous to $\bar{N}_{\text {eff }}$ for border atoms; $\bar{N}_{\text {eff }}^{\text {out }}$ is the same for the outer border atoms, and $\bar{N}_{\text {eff }}^{\text {inn }}$ for the inner border atoms.

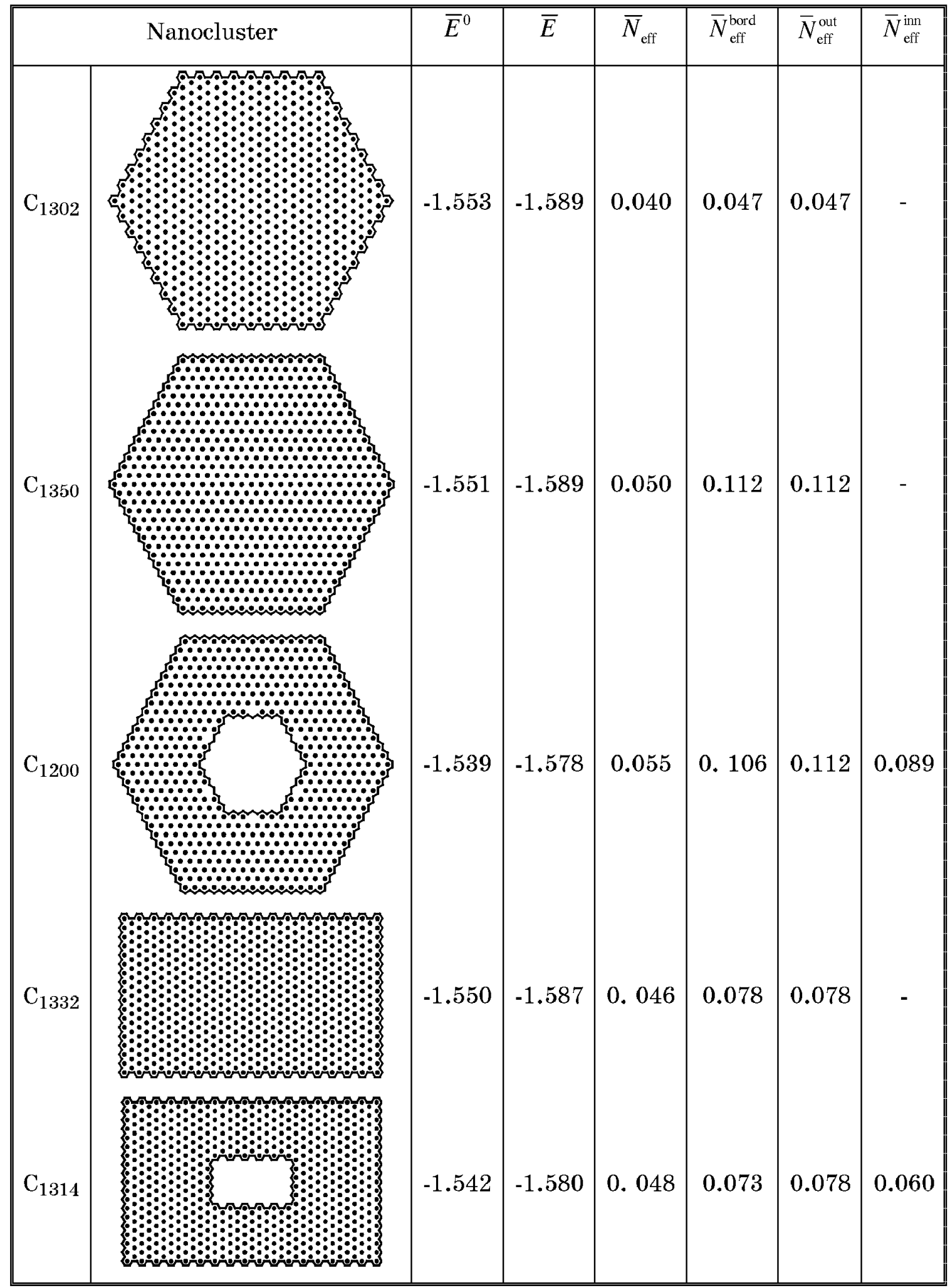


in EUE characteristics. In the zigzag nanoclusters $\left(\mathrm{C}_{1350}\right.$ and $\mathrm{C}_{1200}$ in Table 4$)$ more electrons are unpaired, and these unpaired electrons are preferentially localized on border (edge) atoms. The similar picture was observed in small clusters studied in [17, 18]. The last two systems in Table 4 represent the graphene ribbon-like clusters with zigzag edges (but with armchair edges in the lengthwise boundary). In these clusters the EUE effects are also more pronounced than in its hexagonal armchair structure (such as $\mathrm{C}_{1302}$ in Table 4). The presence of a pore enhances the EUE effects (as in $\mathrm{C}_{1200}$ ). Notice that a moderate irregularity of the edge structure leads to small difference, but details will not be discussed here because of lack of space. Based on the EUE localization index $\bar{N}_{\text {eff }}^{\text {bord }}$ (sum of atomic EUE occupancies divided by a number of the border atoms) we can state that the zigzag edge atoms more unstable, or more reactive, than the armchair edge atoms, and thereby the armchair nanoflakes more stable in full accordance with experiment ([55], p. 382). The main chemical features of the boundary atoms in graphene molecules are recently clarified in papers [56] (their analysis takes off from the idea of Coulson about pseudocarbene and pseudo-carbine types of edge atoms). The final conclusions in [56] are also in line with [55], and with our consideration of the EUE characteristics as specific indicators of the chemically active boundary atoms.

As the last application of the EUE indices we briefly consider the behavior of graphene nanoclusters in strong magnetic fields. For small $\pi$-systems the related problem was recently considered in [57] but without the detailed EUE analysis. Here we study the hexagonal nanoflake $\mathrm{C}_{1350}$ and the nanoflake $C_{1200}$ with a single pore (i.e., the second and third systems in Table 4). In our computations the conventional London gauge-invariant orbitals [58-60] are used within TB (in fact, London's approximation is equivalent to the so-called Peierls substitution [61]). It is important that in the London-Peierls approach the magnetic field modifies only hopping integrals. So, block structure (7) is unchanged, and most of our expressions, such as Eqs. (14)-(16), hold true (about alternant $\pi$-systems in magnetic fields see also [4]). The obtained results for the magnetic-field dependence of the specific energy index $\bar{E}(\bar{E}==0$ in the zero field point) and the EUE index $\bar{N}_{\text {eff }}$ are displayed in Table 5. We see that the electron
Table 5. Dependence of the relative energy $\bar{E}$ and the EUE index $\bar{N}_{\text {eff }}$ on the magnetic field $H$ in the hexalonal graphene nanoflake $\mathrm{C}_{1350}$ and its nanopore $\mathrm{C}_{1200}$ within the QCTB scheme.

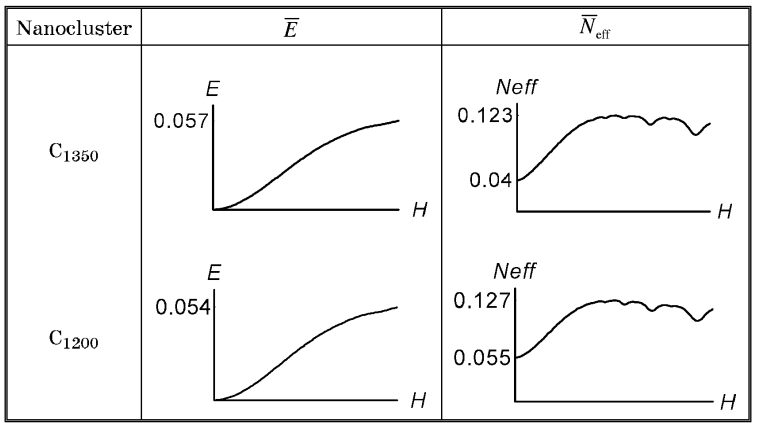

unpairing and the diamagnetism (increasing the specific energy $\bar{E}$ in the field) are significantly increased in the nanoflakes under the strong field. And again, the role of the boundary is also important as computations of the bond currents $I_{\mu \nu}$ (see [60] for definition) show it. We find that $I_{\mu v}$ max, i.e. the maximum of $I_{\mu \nu}$, corresponds to the bond $\mu-v$ situated on the boundary. Specifically, $I_{\mu \nu} \max =0.90$ for $\mathrm{C}_{1350}$, and $I_{\mu \nu} \max =0.92$ for $C_{1200}$ (all values are given in the units of ring current in the benzene). Interestingly, in the latter system the maximal currents are attained on the inter boundary which forms the pore.

\section{Conclusions}

In this work, we proposed a very simple electronic model for bipartite lattices which frequently occur in practice. The method is based on a simple modification of the bipartite graph spectrum (in fact, on the modification of the equivalent Huckel energy spectrum). It allows one to treat the giant covalent networks such as graphenes and use for unpaired electrons the existing energy band spectra within the non-interacting TB framework. The standard diagonalization procedures can be typically applied when analytically derived TB energy spectra cannot be produced. The examples of various nanoflake structures (which are computed here by using a very modest hardware) demonstrate possibilities of the proposed scheme. In the computations we made stress on the EUE characteristics. These cannot be studied in principle with the customary oneelectron TB model, because the unpairing of electrons in singlet states is a purely electron correlation effect. Our calculations of large nanoclusters support the observations, 
which were obtained by others using sophisticated techniques for moderate-size systems, about a difference between the zigzag and armchair graphene molecules. We also examined influence of the external magnetic field which turned out to make a very significant increase of electron unpairing.

In future we will continue to investigate QCTB in various ways. One of the issues is a choice of parameter $\delta$ in the effective Hamiltonians from Eqs. (10, 11). Evidently, it is necessary to seek a more systematic method than used here and based on a rather primitive fitting scheme. Further, there are additional, almost trivial, possibilities for extending QCTB to the bipartite lattices where one of the sublattices is occupied by one type of atom and the other sublattice is occupied by another type of atom (as in the white graphite). It is also worth examining the behavior of large graphene nanoclusters in strong nonstationary fields, and QCTB can serve as a possible starting tool for handling simply and quickly the such challenging problems.

\section{References}

1. C.A.Coulson, G.S.Rushbrooke, Proc.Cambridge Phil. Soc., 36, 139 (1940).

2. A.Brickstock, J.A.Pople, Trans. Farad.Soc., 59, 901 (1954).

3. P.Kasteleyn, Graph Theory and Theoretical Physics, Academic Press, London (1967).

4. A.D.McLachlan, Mol.Phys., 4, 49 (1961).

5. E.H.Lieb, D.C.Mattis, J.Math.Phys., 3, 749 (1962); Z.H.Lieb, Phys.Rev.Lett., 62, 1201 (1989).

6. F.Harary, Graph Theory, Addison-Wesley, Reading, Mass. (1972); D.M.Cvetkovic', M.Doob, H.Sachs, Spectra of Graphs, Theory and Application, Academic Press, New York (1980).

7. Computational Methods for Large Systems: Electronic Structure Approaches for Biotechnology and Nanotechnology, ed. by J.R.Reimers, Wiley, Oxford (2011).

8. W.Matthew, C.Foulkes, R.Haydock, Phys. Rev. $B, 39,12520$ (1989).

9. T.Yamamoto, T.Noguchi, K.Watanabe, Phys. Rev. B, 74, 121409 (2006).

10. S.Reich, J.Maultzsch, C.Thomsen, Phys. Rev. B, 66, 035412 (2002); T.B.Boykin, J.Comput. Electr., 8, 142 (2009); T.B.Boykin, M.Luisier, G.Klimeck et al., J.Appl.Phys., 109, 104304 (2011).

11. M.Damnjanovic, I.Milosevic, Line Groups in Physics: Theory and Applications to Nanotubes and Polymers, Springer, Heidelberg (2011).

12. V.V.Ivanov, I.P.Kisil, A.V.Luzanov, J.Struct. Chem., 37, 537 (1996).
13. K.Takatsuka, T.Fueno, K.Yamaguchi, Theor. Chim.Acta, 48, 175 (1978); V.N.Staroverov, E.R.Davidson, Chem.Phys.Lett., 330, 161 (2000); R.C.Bochicchio, L.Lain, A.Torre, Chem. Phys. Lett., 374, 567 (2003).

14. M.Head-Gordon, Chem. Phys. Lett., 380, 488 (2003).

15. A.V.Luzanov, O.A.Zhikol, Int.J.Quant. Chem., 104, 167 (2005); A.V.Luzanov, O.V.Prezhdo, J.Chem.Phys., 124, 224109 (2006).

16. D.Stuck, T.A.Baker, P.Zimmerman et al., $J$. Chem. Phys., 135, 194306 (2011); K.Yoneda, M.Nakano, K.Fukuda et al., J.Phys.Chem. Lett., 3, $3338 \quad$ (2012); W.Mizukami, Y.Kurashige, T.Yanai, J.Chem. Theor.Comp., 9, 401 (2012); A.V.Luzanov, Int.J.Quant. Chem., 113, 2489 (2013); Z.-h.Cui, H.Lischka, H.Z.Beneberuet et al., J.Am.Chem.Soc., 136, 12958 (2014).

17. F.Plasser, H.Pasalic, M.H.Gerzabek et al., Angew. Chem.Int. Ed., 52, 2581 (2013).

18. A.V.Luzanov, Zh.Strukt.Khim., 55, 845 (2014).

19. D.Casanova, J.Comput.Chem., 35, 944 (2014).

20. A.T.Amos, G.G.Hall, Proc.Roy.Soc.A, 263, 483 (1961).

21. A.Szabo, N.S.Ostlund, Modern Quantum Chemistry, Dover, New York (1996).

22. P.M.Kozlowski, P.Pulay, Theor.Chem.Acc., 100, 12 (1998).

23. K.Ohta, J.Mol.Struct.(Theochem), 587, 33 (2002).

24. M.M.Mestechkin, Theor.Experim.Chem., 2, 327 (1966); A.V.Luzanov, M.M.Mestechkin, Y.B.Vysotskii, Zh.Strukt. Khim., 12, 289 (1971).

25. H.Fukutome, Int.J.Quantum Chem., 20, 955 (1981).

26. G.Kondayya, A.Shukla, Computer Phys. Comm., 183, 677 (2012).

27. G.G.Hall, Proc. Roy.Soc.A, 229, 251 (1955).

28. P.A.M.Dirac, Proc.Cambr.Phil.Soc., 30, 150, 1934; A.S.Davydov, Quantum Mechanics, Pergamon Press, Oxford (1965) [see Eqs. (61.12) and (61.29)].

29. M.M.Mestechkin, Density Matrix Method in the Theory of Molecules, Naukova Dumka, Kiev (1977) [in Russian].

30. N.J.Higham, Functions of Matrices: Theory and Computation, SIAM, Philadelphia, PA (2008).

31. M.M.Mestechkin, Theor.Experim.Chem., 1, 388 (1965).

32. A.A.Ovchinnikov, I.I.Ukrainskii, G.F.Kvenzel', Sov.Phys.Uspekhi, 15, 575 (1973).

33. J.C.W.Chien, Polyacetylene: Chemistry, Physics and Materials Science, Academic Press, Orlando (1984).

34. W.Langer, M.Plischke, D.Mattis, Phys.Rev. Lett., 23, 23, 1448 (1969); W.Langer, D.Mattis, Phys.Lett.A, 36, $139 \quad$ (1971); N.Tyutyulkov, J.Quantum Chem., 9, 683 (1975). 
35. J.G.Pedersen, T.G.Pedersen, Phys.Rev.B, 84, 115424 (2011).

36. D.-E.Jiang, X.-Q.Chen, W.Luo, W.A.Shelton, Chem. Phys. Lett., 483, 120 (2009).

37. J.E.Lennard-Jones., Proc.Roy.Soc.A, 158, 280 (1937).

38. C.A.Coulson, Proc. Roy.Soc.A, 60, 257 (1948).

39. A.V.Luzanov, J.Struct Chem., 43, 711 (2002).

40. J.Delhalle, J.M.Andre, S.Delhalle et al., $J$. Chem.Phys., 60, 595 (1974); J.Delhalle, S.Delhalle, Int.J.Quantum Chem., 11, 349 (1977); O.V.Yazyev, K.N.Kudin, G.E.Scuseria, Phys. Rev.B, 65, 205117 (2002).

41. Graphene Chemistry: Theoretical Perspectives, ed. by D.E.Jiang \& Z.Chen, Wiley, Puerto Rico (2013).

42. I.A.Misurkin, A.A.Ovchinnikov, Mol.Phys., 27, 237 (1974); I.A.Misurkin, A.A.Ovchinnikov, Russ. Chem. Rev., 46, 96 (1977).

43. A.M.S.Macedo, M.C.Dos Santos, M.D.Coutinho-Filho, C.A.Macedo, Phys.Rev. Lett., 74, 1851 (1995).

44. D.E.Jiang, B.G.Sumpter, S.J.Dai, Chem. Phys., 126, 134701 (2007); M.Das, J.Chem. Phys., 140, 124317 (2014).

45. S.V.Vonsovskii, M.S.Svirskii, JETP, 30, 140 (1970); S.V.Vonsovsky, Magnetism, Vol. 2, Wiley, New York (1974).

46. A.V.Luzanov, O.V.Prezhdo, J.Chem.Phys., 135, 094107 (2011).

47. A.K.Geim, K.S.Novoselov, Nature Mater., 6, 183 (2007).

48. Introduction to Graphene-Based Nanomaterials from Electronic Structure to Quantum Transport, ed. by L.E.F. Foa Torres, S.Roche, J.-C.Charlie, Cambridge University Press (2014).
49. E.L.Wolf, Graphene: a New Paradigm in Condensed Matter and Device Physics, Oxford, Oxford University Press (2014).

50. X.Zhang, J.Xin, F.Ding, Nanoscale, 5, 2556 (2013).

51. Z.Luo, S.Kim, N.Kawamoto et al., ACS Nano, 5, 9154 (2011).

52. A.S.Barnard, I.K.Snook, Modell.Simul. Mater. Sci.Eng., 19, 054001 (2011).

53. J.Fernandez-Rossier, J.J.Palacios, Phys.Rev. Lett., 99,177204 (2007); V.Barone, O.Hod, G.E.Scuseria, Nano Lett., 6, 2748 (2006); X.Jia, J.Campos-Delgado, M.Terrones et al., Nanoscale, 3, 86 (2011); C.Cocchi, D.Prezzi, A.Ruini et al., J.Phys.Chem.C, 116, 17328 (2012); O.S.Karpenko, V.V.Lobanov, N.T.Kartel, Chem.Phys. Technol.Surf., 4, 123 (2013).

54. F.Ouyang, Z.Yang, J.Xiao et al., J.Phys. Chem.C, 114, 15578 (2010); M.R.Philpott, Y.Kawazoe, J.Chem.Phys., 134, 124706 (2011).

55. A.Cresti, N.Nemec, B.Biel et al., Nano Research, 1, 361 (2008).

56. L.R.Radovic, B.Bockrath, J.Am.Chem.Soc., 127, 5917 (2005); L.R.Radovic, A.F.Silva-Villalobos, A.B.Silva-Tapia, F.Vallejos-Burgos, Carbon, 49, 3471 (2011).

57. A.V.Luzanov, Zh.Strukt.Khim., $\quad \mathbf{5 4 ,} 277$ (2013).

58. F.London, J.Phys. Rad., 8, 397 (1937).

59. J.W.Emsley, J.Feeney, L.H.Sutcliffe, HighResolution NMR Spectroscopy, Pergamon, London (1965).

60. A.Ceulemans, L.F.Chibotaru, P.W.Fowler, Phys. Rev. Lett., 80, 1861 (1998).

61. R.Peierls, Z. Phys., 80, 763 (1933). 